OPEN ACCESS

Edited by:

Jae-Ho Shin,

Kyungpook National University,

South Korea

Reviewed by:

Hidetoshi Urakawa,

Florida Gulf Coast University, USA

Ji-Hoon Lee,

Chonbuk National University,

South Korea

*Correspondence:

Lone Gram

gram@bio.dtu.dk

Specialty section:

This article was submitted to

Evolutionary and Genomic

Microbiology,

a section of the journal

Frontiers in Microbiology

Received: 29 October 2016

Accepted: 27 February 2017

Published: 13 March 2017

Citation:

Machado H, Cardoso J, Giubergia S, Rapacki K and Gram L (2017) FurlOS: A Web-Based Tool for Identification of Vibrionaceae

Species Using the fur Gene.

Front. Microbiol. 8:414.

doi: 10.3389/fmicb.2017.00414

\section{FurlOS: A Web-Based Tool for Identification of Vibrionaceae Species Using the fur Gene}

\author{
Henrique Machado ${ }^{1,2}$, João Cardoso², Sonia Giubergia ${ }^{1,2}$, Kristoffer Rapacki $^{3}$ and \\ Lone Gram ${ }^{\text {* }}$
}

${ }^{1}$ Department of Biotechnology and Biomedicine, Technical University of Denmark, Kongens Lyngby, Denmark, ${ }^{2}$ The Novo Nordisk Foundation Center for Biosustainability, Technical University of Denmark, Kongens Lyngby, Denmark, ${ }^{3}$ Center for Biological Sequence Analysis, Department of Bioinformatics, Technical University of Denmark, Kongens Lyngby, Denmark

Gene based methods for identification of species from the Vibrionaceae family have been developed during the last decades to address the limitations of the commonly used $16 S$ rRNA gene phylogeny. Recently, we found that the ferric-uptake regulator gene (fur) can be used as a single identification marker providing species discrimination, consistent with multi-locus sequencing analyses and whole genome phylogenies. To allow for broader and easy use of this marker, we have developed an online prediction service that allows the identification of Vibrionaceae species based on their fur-sequence. The input is a DNA sequence that can be uploaded on the web service; the output is a table containing the strain identifier, e-value, and percentage of identity for each of the matches with rows colored in green for hits with high probability of being the same species. The service is available on the web at: http://www.cbs.dtu.dk/services/ furlOS-1.0/. The fur-sequences can be derived either from genome sequences or from PCR-amplification of the genomic region encoding the fur gene. We have used 191 strains identified as Vibrionaceae based on 16S rRNA gene sequence to test the PCR method and the web service on a large dataset. We were able to classify 171 of 191 strains at the species level and 20 strains remained unclassified. Furthermore, the fur phylogenetics and subsequent in silico DNA-DNA hybridization demonstrated that two strains (ATCC 33789 and ZS-139) previously identified as Vibrio splendidus are more closely related to $V$. tasmaniensis and $V$. cyclitrophicus, respectively. FurlOS is an easyto-use online service that allows the identification of bacteria from the Vibrionaceae family at the species level using the fur gene as a single marker. Its simplistic design and straightforward pipeline makes it suitable for any research environment, from academia to industry.

\section{Keywords: Vibrionaceae, Vibrio, Photobacterium, fur gene, phylogeny, identification}

\section{INTRODUCTION}

A key aspect in microbial taxonomy is the identification of microorganisms at the species or genus level. This is important to distinguish pathogenic species in health and environmental sciences, to identify beneficial or symbiotic species and also to study microbial diversity in environmental niches in Nature. Due to the widespread use and importance of bacterial identification, methods and processes should be accurate, affordable, fast and easy to use (Urakawa et al., 1997; Amaral et al., 2014). 
The Vibrionaceae is a large family of marine Gram-negative Gammaproteobacteria, which includes organisms of different environmental importances, e.g., symbiotic (e.g., Vibrio fischeri), bioactive (e.g., V. coralliilyticus and Photobacterium galatheae), and pathogenic organisms (e.g., V. cholerae and Alivibrio salmonicida). Several of the human pathogenic Vibrio species, such as $V$. cholerae and $V$. parahaemolyticus, are mesophilic organisms and changes in sea water temperature and salinity influence their distribution (Reid et al., 2011; Huehn et al., 2014; Le Roux et al., 2015; Xu et al., 2015). The increase in sea water temperature has been linked to a number of human illness outbreaks caused by Vibrio species (e.g., V. cholerae, $V$. parahaemolyticus, V. vulnificus) (Le Roux et al., 2015) and to several epidemics in marine animals, such as oysters, salmon, sea bass, eel, trout, shrimps, and corals, caused by $V$. salmonicida, $V$. anguillarum, $P$. damselae, $V$. vulnificus, and $V$. coralliilyticus (Ottaviani et al., 2012; Huehn et al., 2014; Le Roux et al., 2015). The rising sea water temperatures have also been linked to the increase in the numbers of $V$. cholerae associated with plankton in the North Sea (Vezzulli et al., 2012). The increased spread of Vibrio pathogens and their importance as infectious and/or food poisoning agents has a direct impact on health of mankind and requires changes in microbiological food control processes and clinical settings (Nair et al., 2007). Any survey or intervention to reduce risk requires that the organisms can be rapidly and correctly identified.

Identification of species from the Vibrionaceae family has primarily been based on Multi-Locus Sequencing Analysis (MLSA), which relies on the amplification and sequencing of up to nine genes ( $f t s Z, g a p A, g y r B, m r e B, p y r H, r e c A, r p o A$, top $A$, and the 16S rRNA gene) (Thompson et al., 2005; Sawabe et al., 2007, 2013; Gabriel et al., 2014). This has been necessary due to the limitations of 16S rRNA gene phylogeny in this family, an issue addressed with coupling of other techniques such as restriction fragment length polymorphism analyses (Urakawa et al., 1997, 1999), but ultimately unsolvable due to several (7-15) different copies of 16S rRNA gene encoded in a single genome (Reen et al., 2006; Jensen et al., 2009; Machado and Gram, 2015). We have recently identified the ferric-uptake regulator gene (fur) as a new phylogenetic marker in the Vibrionaceae family, and developed a PCR based method for the amplification of the genomic region encoding the fur gene (Machado and Gram, 2015). Its use as a single phylogenetic marker in the classification of Vibrionaceae at the species level could reduce the timing and cost of strain identification. To enable the use of this potential worldwide, we here describe the design of an online platform, FurIOS 1.0, which allows an easy and fast identification of Vibrionaceae species using only their fur DNA sequence. We also use a collection of 191 Vibrionaceae strains to demonstrate its potential.

\section{MATERIALS AND METHODS}

\section{Database Design}

A fur gene sequences database was created by extracting the sequences from available whole genome sequences and using the data collected during the design of the gene amplifi- cation methodology (Machado and Gram, 2015). The BLAST compatible version was generated using makeblastdb from NCBI BLAST + command line tools.

\section{Implementation of FurIOS $\mathbf{1 . 0}$}

FurIOS is implemented in Python, compatible with version 2. The BLAST is performed using NCBI BLAST + version (2.2.28+) (Tatusova and Madden, 1999), blastn calls and output parsing is handled using BioPython (Cock et al., 2009). The script verifies the format of the input, parameterizes the blastn command call and formats the output. BLAST runs with the following parameters: $e$-value of 10 ; gap open penalty of 5 ; gap extension penalty of 2 ; mismatch penalty of -3 ; match reward of 2 ; word size of 11 ; maximum number of returned alignments of 50 . These parameters are the default CLC Main Workbench (CLC Aarhus, Denmark version 7) used in the design of the method and evaluation of the potential of the fur as a phylogenetic marker (Machado and Gram, 2015). The web interface is provided by the Center for Biological Sequence Analysis (CBS), Technical University of Denmark and the webpages are designed according to their standards. Documentation can be also found on the website.

\section{Testing of the Identification Pipeline Bacterial Strains and Genomic DNA Extraction}

The bacterial strains used in the implementation of the identification pipeline were 191 Vibrionaceae strains from the Galathea 3 culture collection (Gram et al., 2010). These strains have been identified as Vibrionaceae by analysis of the $16 \mathrm{~S}$ rRNA gene sequence (Gram et al., 2010). Here, we aimed at a more specific classification, at the species level. Strains were grown overnight at $25^{\circ} \mathrm{C}$ and 200 r.p.m. in Marine Broth (Difco 279110), before genomic DNA was extracted using the NucleoSpin ${ }^{\circledR}$ Tissue Kit (Macherey-Nagel, Düren, Germany). Genomic DNA quality was checked by $1 \%$ agarose gel electrophoresis and quantified by absorbance using DeNovix DS-11 (DeNovix Inc., Wilmington, DE, USA).

\section{PCR Amplification and Sequencing}

Amplification of the fur gene was performed as previously described (Machado and Gram, 2015), with few changes. Briefly, amplifications were performed in a total volume of $25 \mu \mathrm{L}$ using $5 \mathrm{ng}$ final concentration of genomic DNA as template, $0.2 \mu \mathrm{M}$ of each primer [fur_AP_fw and fur_AP_rv (Machado and Gram, 2015)] and TEMPase Hot Start Master Mix Blue (Ampliqon A/S, Odense, Denmark), following the producers instructions. The PCR amplification was carried out in a thermal cycler (Applied Biosystems ${ }^{\circledR}$ Veriti $^{\circledR}$ 96-Well Thermal Cycler) as follows: $15 \mathrm{~min}$ initial denaturation step at $95^{\circ} \mathrm{C}$, followed by 30 cycles of $95^{\circ} \mathrm{C}$ for $25 \mathrm{~s}, 52^{\circ} \mathrm{C}$ for $25 \mathrm{~s}$, and $72^{\circ} \mathrm{C}$ for $1 \mathrm{~min}$, with a final extension step of $5 \mathrm{~min}$ at $72^{\circ} \mathrm{C}$. The amplified products were visualized after agarose gel electrophoresis (1\%) and ethidium bromide staining. The PCR products were enzymatically purified by treatment with Exonuclease I (ExoI) (Thermo Scientific) and FastAP Thermosensitive Alkaline Phosphatase (Thermo Scientific) before being sequenced by Macrogen (Macrogen Europe, Amsterdam, The Netherlands). The sequencing reads 


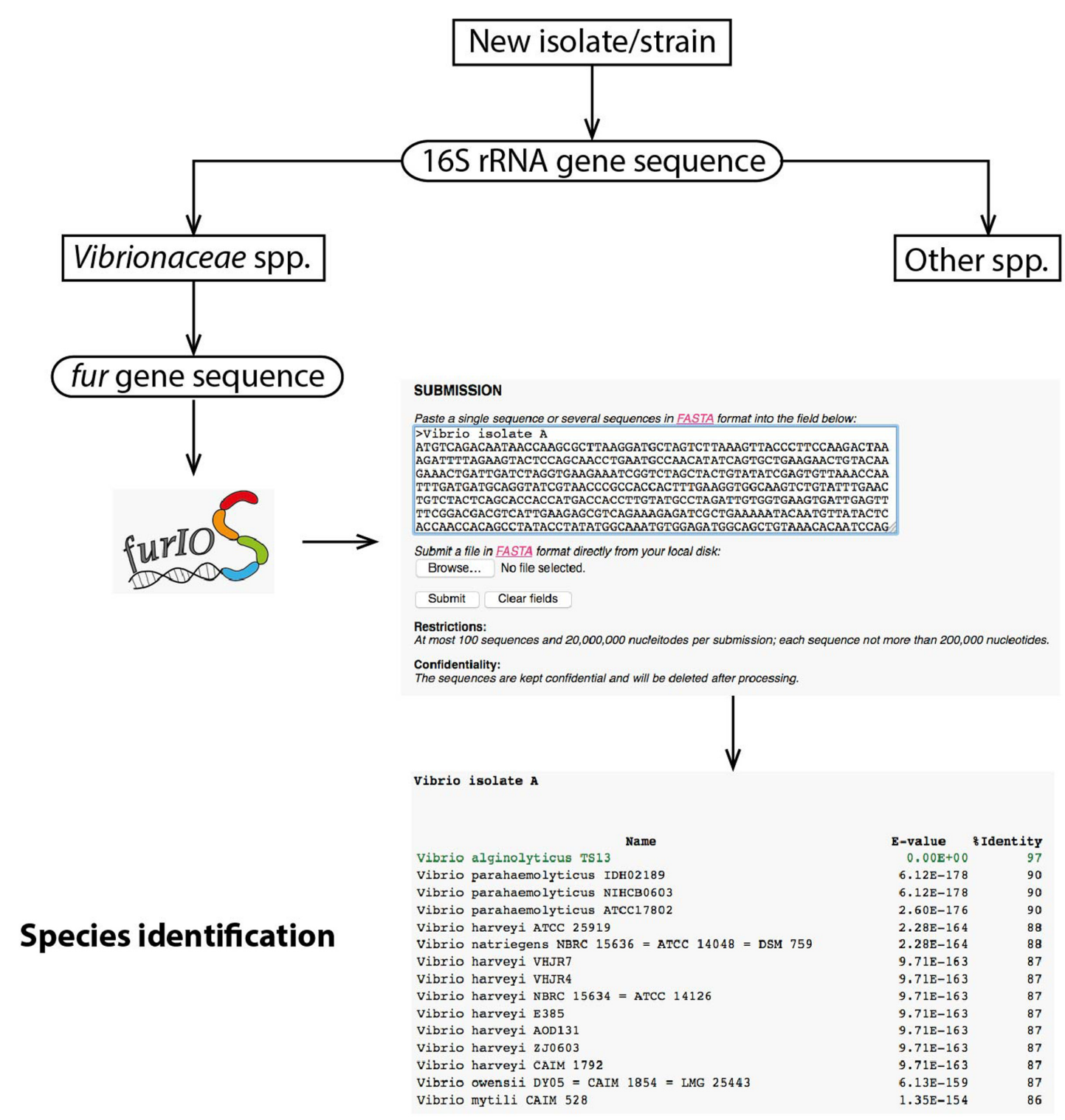

FIGURE 1 | Workflow and visualization example. Example of sequence submission, this should be in FASTA format and can be a multiple sequence submission. Example of a result in table format, which includes the $E$-value and the percentage of identity; the predicted species is colored in green.

were assembled and analyzed using CLC Main Workbench (CLC Aarhus, Denmark version 7).

\section{Species Identification}

The fur gene sequences obtained from the PCR and sequencing reactions were merged into a single FASTA file. This file contained 191 fur sequences, each with a headline identifying the strain number (e.g., ">S2757"). This file was uploaded to the web interface of the FurIOS $1.0^{1}$ and the analyses performed. The highest hit was selected from the output for each of the strains (Supplementary Table S1) and the strain classified at the species level if the percentage of identity was above $95 \%$. In cases where

${ }^{1}$ http://www.cbs.dtu.dk/services/furIOS-1.0/ lower than 95\% identity was obtained, the strain was classified as a possible new species. The fur gene sequences have been deposited on GenBank under accession numbers KU756296 - KU756481, KP721394, KP721390, KP721391, KP721399, and KP721400.

\section{Genomic Analyses of Vibrio splendidus Strains}

In silico DNA-DNA hybridization was performed for $V$. splendidus strains representing three different fur phylogenetic clusters. The genomes were compared using the Genome-toGenome Distance Calculator 2.1 (GGDC) tool from DSMZ 2 (Auch et al., 2010a,b; Meier-Kolthoff et al., 2013). The used

\footnotetext{
${ }^{2}$ http://ggdc.dsmz.de/
} 


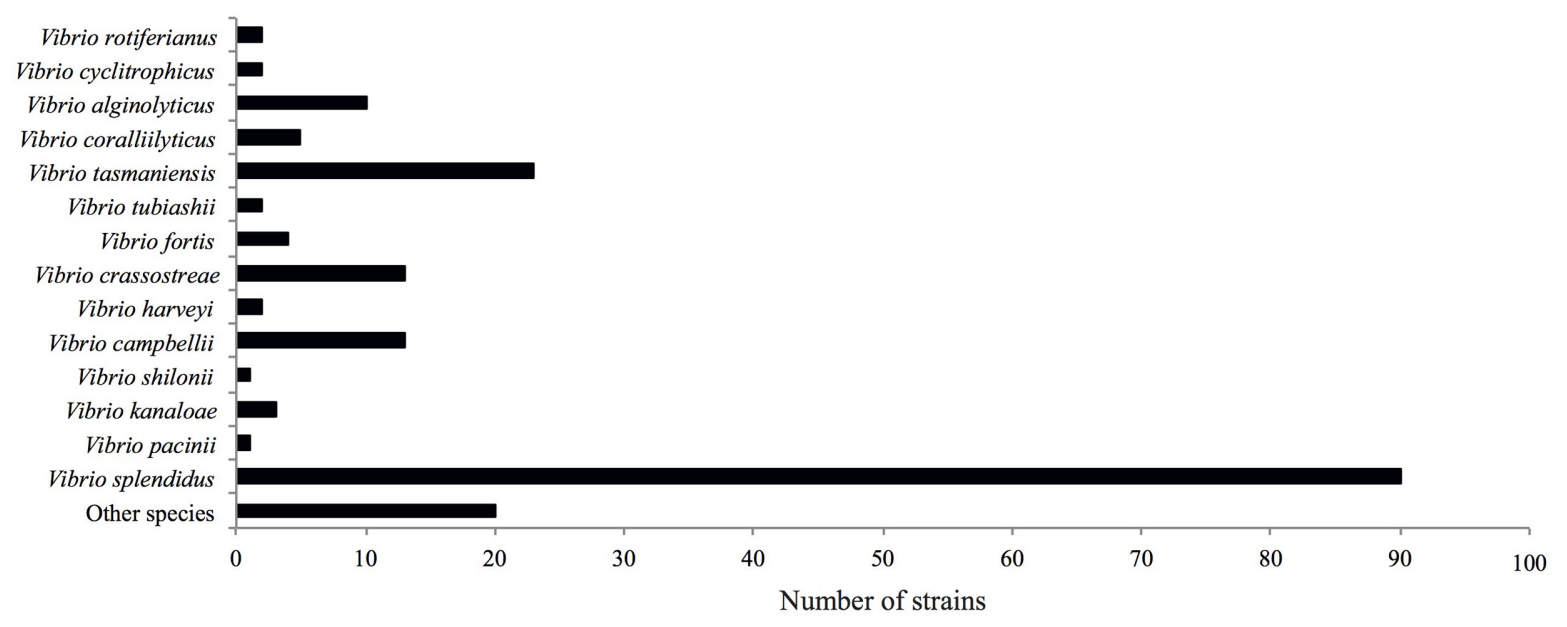

FIGURE 2 | Classification of 191 strains belonging to the Galathea 3 Vibrionaceae culture collection (Gram et al., 2010). The identification corresponds to the highest hit obtained from the analyses using FurlOS 1.0. In cases where lower than 95\% identity was obtained, the strain was classified as "other species."

whole genome sequences of $V$. splendidus strains FF-500, 1F-157, FF-6, ZF-90, 1S-124, ZS-139, ATCC 33789, and NCCB $53037^{\mathrm{T}}$ are publically available at NCBI under whole genome sequenced accession numbers AJZH00000000, AJZJ00000000, AJZI00000000, AJZF00000000, AJZL00000000, AJZE00000000, AFWG00000000, LNQX00000000, respectively.

\section{RESULTS}

\section{Database Design and Implementation}

The designed database includes 134 sequences representing 78 species of the Vibrionaceae family, covering its six genera (Vibrio, Photobacterium, Aliivibrio, Grimontia, Enterovibrio, and Salinivibrio). This includes whole the available fur sequences, weather individual or retrieved from whole genome sequences.

Users can access FurIOS via the web interface: http://www. cbs.dtu.dk/services/furIOS-1.0/. The input can be the whole fur open-reading frame of the sequence of the strain to be identified, the whole sequenced fragment, whole genome sequences or metagenomics data (with a maximum of 100 contigs of up to 200,000 nucleotides, making a total of 20 million nucleotides per submission). Submission of untreated sequences (raw sequencing data) is possible and decreases the need for sequence processing by the user, thereby accelerating the identification process. The sequences can be uploaded on the web service by "copy and paste" or using a file in FASTA format (Figure 1). The output is a table containing the strain identifier, $e$-value, and percentage of identity for each of the matches with rows colored in green for hits with percentage of identity higher than 95\% (Figure 1), representing a high probability of being the same species (Machado and Gram, 2015). The service is provided with pre-established settings used in the design and evaluation of fur as a phylogenetic marker (Machado and Gram, 2015), however, a portable version where parameters can be selected by the user will be provided upon request.

\section{Testing of the Identification Pipeline}

The identification pipeline from isolate to species identification was used in the classification of the Vibrionaceae isolates from the Galathea 3 culture collection (Gram et al., 2010). These strains have been previously identified as Vibrionaceae by $16 \mathrm{~S}$ rRNA gene sequence analysis (Gram et al., 2010). Here, we aimed at a more specific classification, at the species level. Therefore, genomic DNA isolation followed by fur gene amplification and sequencing was performed in 191 Vibrionaceae strains.

The fur gene sequences obtained were analyzed using FurIOS 1.0. Species affiliation could be assigned to 171 of the 191 strains (Figure 2). Based on the fur sequence, the remaining 20 isolates had an identity lower than $95 \%$ and were therefore classified as "other species" (Figure 2). These 20 strains could potentially be new Vibrio species or species not yet represented in the FurIOS database, due to unavailability of whole genome or fur sequences. Of the 20 "other species," 15 were 90-94\% similar to the fur gene sequences of known species, whereas the remaining five strains had similarities between 82 and 89\% (Supplementary Table S1). From the phylogenetic analysis it is also possible to evaluate the relatedness of some of the strains identified as "other species" (Figure 3). Some of these will most likely be the same species (e.g., S1348, S1349, and S1350, or S2320, S2321, and S2322).

\section{Genomic Analyses of Vibrio splendidus Strains}

Ninety of the 171 strains were identified as V. splendidus, however, these did not cluster as one tight single cluster but were distributed across the phylogenetic tree (Figure 3). This species was represented in three clusters, two large and one small, all placed within the Splendidus clade, to which $V$. fortis, $V$. cyclitrophicus, $V$. crassostreae, $V$. tasmaniensis, and $V$. kanaloae species also belong (Sawabe et al., 2013). The three V. splendidus clusters correspond to fur homology to different $V$. splendidus strains. The first cluster contained strains with fur homology to V. splendidus strains FF-6, FF-500, 1F-157, 1S-124, and ZF-90 


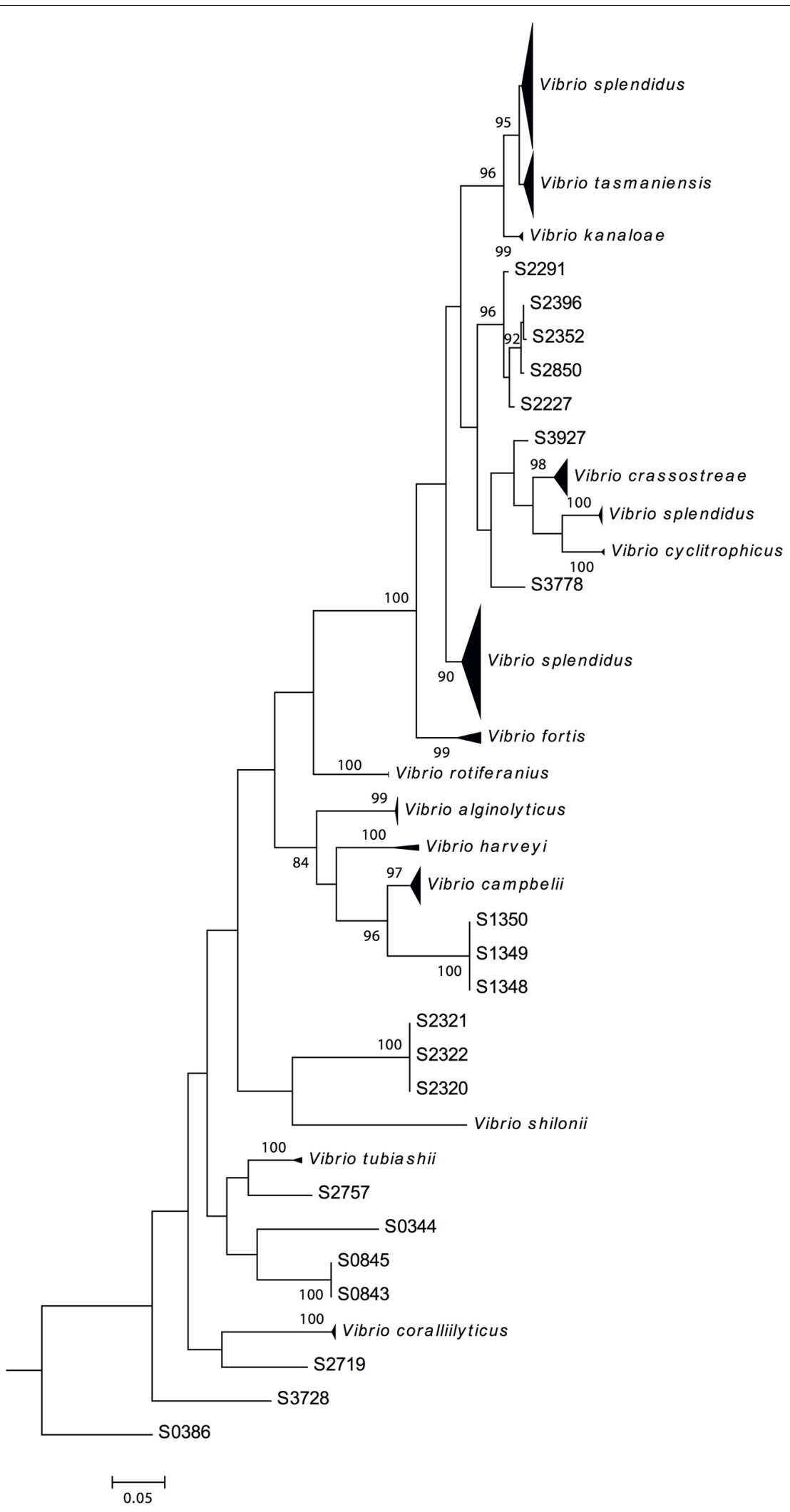

FIGURE 3 | Phylogenetic tree of 191 Galathea 3 Vibrionaceae strains. The tree is based on the complete fur gene sequences analysis and was constructed by the neighbor-joining method. S0386 was used as the outlier, since it classified as a Photobacterium sp. The nodes with bootstrap support of 70 or more are indicated (1000 replications). 
(Supplementary Table S1). The second smaller cluster and the third cluster contained strains with fur homology to V. splendidus ZS-139 and ATCC 33789, respectively. The different clustering suggests a different phylogenetic relationship between these strains, previously identified as the same species.

These discrepancies have been previously reported and attributed to the possible misidentification of Vibrio strains (Gomez-Gil, 2004; Thompson et al., 2007; Lin et al., 2010) or to the genetic diversity and polyphyletic nature of $V$. splendidus (Thompson and Hoste, 2001; Thompson et al., 2005; Pascual et al., 2010). To address this, in silico DNA-DNA hybridization was performed for strains of the $V$. splendidus representing the three different fur phylogenetic clusters of this species. Based on DNA-relatedness (Table 1), strains ATCC 33789 and ZS-139 were not similar enough to $V$. splendidus type-strain NCCB $53037^{\mathrm{T}}$ to be considered the same species. These possibly represent a new Vibrio species. The fur sequences with high homology to the sequence from strain ZS-139 were closely related to V. cyclitrophicus (93\% identity) while homology to strain ATCC 33789 placed them phylogenetically close to $V$. tasmaniensis $(91 \%$ identity).

\section{DISCUSSION}

FurIOS is an easy-to-use online service that allows the identification of bacteria from the Vibrionaceae family at the species level using the fur gene as a single identification marker. This online service available to any user worldwide is an extra effort to implement the use of the recently developed method for the amplification of the fur gene sequence, with greater discriminatory power when compared to MLSA or 16S rRNA analyses. Here, we have applied this tool in the classification of environmental Vibrionaceae strains belonging to the global culture collection Galathea 3.

We identified at the species level $90 \%$ of the 191 Vibrionaceae strains from the Galathea 3 culture collection. The most abundant species was Vibrio splendidus, followed by $V$. tasmaniensis, $V$. crassostreae, and V. campbellii (Figure 2). The collection was based on culturing from marine samples and subsequent testing of antibacterial activity against the fish pathogen $V$. anguillarum
(Gram et al., 2010), and this may explain the over-representation (70\%) of the species belonging to the Splendidus clade (Sawabe et al., 2013). Several studies have shown the presence of $V$. splendidus in water samples through all seasons, with higher predominance in summer, and in locations ranging from the arctic to the tropics (Thompson et al., 2004; Jensen et al., 2009). These features reflect the great adaptability of this species (Jensen et al., 2009), which can also explain its over-representation in a global culture collection such as the Galathea 3 collection. The analysis also provided evidence that two $V$. splendidus strains ATCC 33789 and ZS-139 are indeed not V. splendidus species, which we confirmed using whole genome phylogenetic analysis (Table 1).

Besides the over-representation of strains from the Splendidus clade, this dataset allowed the evaluation of the potential of this identification method and web-tool in the classification of Vibrionaceae environmental samples at the species level, by means of a single PCR reaction and sequencing of its product. The method presented here can be used for identification of Vibrionaceae species in less than $24 \mathrm{~h}$, and it requires minimal equipment and service. The analyses of the 191 sequences using the FurIOS web service took only $3 \mathrm{~min}$, although this time may depend on the server usage at the moment of use. Sequence based identification has in other settings also been developed into online tools, which have been successfully used for several years for MLSA of pathogenic bacteria ${ }^{3,4,5}$. A tool based on MLSA has been under development for identification of Vibrio species ${ }^{6}$, however, it appears not to be functional. FurIOS is therefore the first functional online service developed for the classification of Vibrionaceae species and it is easier to implement and use than the MLSA based analyses, because it uses a single gene with great discriminatory power (Machado and Gram, 2015; Giubergia et al., 2016).

This identification exercise provided 20 isolates with an inconclusive species attribution ("other species" - Figure 2). These might constitute new species or species not yet represented

\footnotetext{
${ }^{3}$ http://mlst.warwick.ac.uk/mlst/

${ }^{4}$ http://www.mlst.net/

${ }^{5}$ http://bigsdb.web.pasteur.fr/

${ }^{6}$ http://www.lge.ibi.unicamp.br/temp/vibrionaceae/
}

TABLE 1 | In silico DNA-DNA hybridization estimate of the different Vibrio splendidus strains.

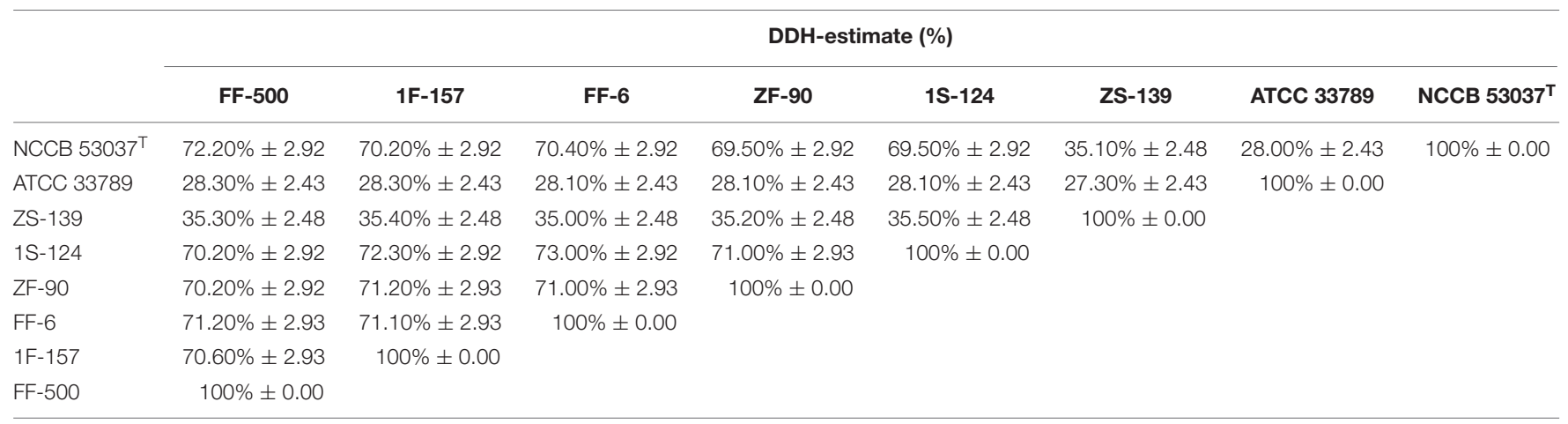

The estimate was performed using the Genome-to-Genome Distance Calculator 2.1 (GGDC) tool from DSMZ. 
in the FurIOS database. Although the number of species represented in the database is lower than the number of species so far described within this family, the use of the fur gene as an identification marker and the publication of more fur sequences will allow the improvement of the database by increasing the number of species represented. Newly published fur sequences can be sent to the corresponding author who will act as a database curator. Also publications making use of FurIOS will be checked for relevant sequences to be added. Supporting the possibility of "other species" being a new species is the case of strain S2757. Its fur gene sequence had an $89 \%$ identity to $V$. tubiashii DSM 19142 and it was therefore here classified as "other species." This strain has been further studied and has been recently described as the type strain of the new species V. galatheae (Giubergia et al., 2016). In that study, 16 S rRNA, MLSA, and fur gene analyses were performed and the fur gene, as a single gene analysis, provided as good discrimination as did the MLSA analysis (Giubergia et al., 2016). This exemplifies the use of the fur gene in the classification of a new species belonging to the Vibrionaceae family.

Although there might not be a perfect single gene for species identification or phylogenetic evaluation, the fur gene seems so far the best "single" gene identified in the classification of Vibrio species and the development of this tool is an effort to bring that to use and help the scientific community with identification of strains from the Vibrionaceae family. Yet, evaluation of specific phylogenetic relationships should be done using multiple genes, such as MLSA analyses. It is not clear why fur mutational rate allows distinction of Vibrionaceae at the species level, but we have observed indications of the same pattern in Pseudoalteromonas (Machado et al., 2015).

The recent findings have provided the Vibrionaceae taxonomy field with new and more accurate approaches to evaluate the phylogeny and taxonomy relatedness between the different species of this family. This comes as a great opportunity to

\section{REFERENCES}

Amaral, G. R. S., Dias, G. M., Wellington-Oguri, M., Chimetto, L., Campeão, M. E., Thompson, F. L., et al. (2014). Genotype to phenotype: identification of diagnostic vibrio phenotypes using whole genome sequences. Int. J. Syst. Evol. Microbiol. 64, 357-365. doi: 10.1099/ijs.0.057927-0

Auch, A. F., Klenk, H.-P., and Göker, M. (2010a). Standard operating procedure for calculating genome-to-genome distances based on high-scoring segment pairs. Stand. Genomic Sci. 2, 142-148. doi: 10.4056/sigs.541628

Auch, A. F., von Jan, M., Klenk, H.-P., and Göker, M. (2010b). Digital DNA-DNA hybridization for microbial species delineation by means of genome-to-genome sequence comparison. Stand. Genomic Sci. 2, 117-134. doi: 10.4056/sigs.531120

Cock, P. J. A., Antao, T., Chang, J. T., Chapman, B. A., Cox, C. J., Dalke, A., et al. (2009). Biopython: freely available Python tools for computational molecular biology and bioinformatics. Bioinformatics 25, 1422-1423. doi: 10.1093/bioinformatics/btp163

Gabriel, M. W., Matsui, G. Y., Friedman, R., and Lovell, C. R. (2014). Optimization of multilocus sequence analysis for identification of species in the genus Vibrio. Appl. Environ. Microbiol. 80, 5359-5365. doi: 10.1128/AEM.01206-14

Giubergia, S., Machado, H., Matei, R. V., and Gram, L. (2016). Vibrio galatheae sp. nov., a member of the family Vibrionaceae isolated from a mussel. Int. J. Syst. Evol. Microbiol. 66, 347-352. doi: 10.1099/ijsem.0.000723

Gomez-Gil, B. (2004). Vibrio hispanicus sp. nov., isolated from Artemia sp. and sea water in Spain. Int. J. Syst. Evol. Microbiol. 54, 261-265. doi: 10.1099/ijs.0. 02775-0 re-evaluate the evolutionary theories in this family as well to discover and correctly classify newly identified species. Regarding FurIOS, its simplistic design and straightforward pipeline makes it suitable for any research environment, from academia to industry, and especially for quick species identification in clinical and food-safety settings, where Vibrionaceae strains are of high risk (e.g., microbiological control of sea food products).

\section{AUTHOR CONTRIBUTIONS}

HM designed the study and the database used, and together with SG performed the testing of the web-based tool. JC carried out the programming and together with KR the implementation of the web-based tool. All authors contributed to the writing of the manuscript. All authors read and approved the final manuscript.

\section{FUNDING}

HM and SG were supported by a Ph.D. grant from the People Programme (Marie Curie Actions) of the European Union's Seventh Framework Programme FP7-People-2012-ITN, under grant agreement No. 317058, "BACTORY." JC acknowledges the support by the Novo Nordisk Foundation through The Novo Nordisk Foundation Center for Biosustainability.

\section{SUPPLEMENTARY MATERIAL}

The Supplementary Material for this article can be found online at: http://journal.frontiersin.org/article/10.3389/fmicb. 2017.00414/full\#supplementary-material

Gram, L., Melchiorsen, J., and Bruhn, J. B. (2010). Antibacterial activity of marine culturable bacteria collected from a global sampling of ocean surface waters and surface swabs of marine organisms. Mar. Biotechnol. 12, 439-451.[ doi: 10.1007/s10126-009-9233-y

Huehn, S., Eichhorn, C., Urmersbach, S., Breidenbach, J., Bechlars, S., Bier, N., et al. (2014). Pathogenic vibrios in environmental, seafood and clinical sources in Germany. Int. J. Med. Microbiol. 304, 843-850. doi: 10.1016/j.ijmm.2014.07.010

Jensen, S., Frost, P., and Torsvik, V. L. (2009). The nonrandom microheterogeneity of 16S rRNA genes in Vibrio splendidus may reflect adaptation to versatile lifestyles. FEMS Microbiol. Lett. 294, 207-215. doi: 10.1111/j.1574-6968.2009. 01567.x

Le Roux, F., Wegner, K. M., Baker-Austin, C., Vezzulli, L., Osorio, C. R., Amaro, C., et al. (2015). The emergence of Vibrio pathogens in Europe: ecology, evolution, and pathogenesis (Paris, 11-12th March 2015). Front. Microbiol. 6:830. doi: $10.3389 /$ fmicb.2015.00830

Lin, B., Wang, Z., Malanoski, A. P., O’Grady, E. A., Wimpee, C. F., Vuddhakul, V., et al. (2010). Comparative genomic analyses identify the Vibrio harveyi genome sequenced strains BAA-1116 and HY01 as Vibrio campbellii. Environ. Microbiol. Rep. 2, 81-89. doi: 10.1111/j.1758-2229.2009.00100.x

Machado, H., and Gram, L. (2015). The fur gene as a new phylogenetic marker for Vibrionaceae species identification. Appl. Environ. Microbiol. 81, 2745-2752. doi: 10.1128/AEM.00058-15

Machado, H., Sonnenschein, E. C., Melchiorsen, J., and Gram, L. (2015). Genome mining reveals unlocked bioactive potential of marine Gram-negative bacteria. BMC Genomics 16:1365. doi: 10.1186/s12864-015-1365-z 
Meier-Kolthoff, J. P., Auch, A. F., Klenk, H.-P., and Göker, M. (2013). Genome sequence-based species delimitation with confidence intervals and improved distance functions. BMC Bioinformatics 14:60. doi: 10.1186/1471-2105-14-60

Nair, G. B., Ramamurthy, T., Bhattacharya, S. K., Dutta, B., Takeda, Y., and Sack, D. A. (2007). Global dissemination of Vibrio parahaemolyticus serotype O3:K6 and its serovariants. Clin. Microbiol. Rev. 20, 39-48. doi: 10.1128/CMR. 00025-06

Ottaviani, D., Leoni, F., Serra, R., Serracca, L., Decastelli, L., Rocchegiani, E., et al. (2012). Nontoxigenic Vibrio parahaemolyticus strains causing acute gastroenteritis. J. Clin. Microbiol. 50, 4141-4143. doi: 10.1128/JCM.01993-12

Pascual, J., Macián, M. C., Arahal, D. R., Garay, E., and Pujalte, M. J. (2010). Multilocus sequence analysis of the central clade of the genus Vibrio by using the $16 \mathrm{~S}$ rRNA, recA, pyrH, rpoD, gyrB, rctB and toxR genes. Int. J. Syst. Evol. Microbiol. 60, 154-165. doi: 10.1099/ijs.0.010702-0

Reen, F. J., Almagro-Moreno, S., Ussery, D., and Boyd, E. F. (2006). The genomic code: inferring Vibrionaceae niche specialization. Nat. Rev. Microbiol. 4, 697-704. doi: 10.1038/nrmicro1476

Reid, P. C., Gorick, G., and Edwards, M. (2011). Climate Change and Marine Ecosystem Research. Plymouth: Sir Alister Hardy Foundation for Ocean Science (SAHFOS).

Sawabe, T., Kita-Tsukamoto, K., and Thompson, F. L. (2007). Inferring the evolutionary history of vibrios by means of multilocus sequence analysis. J. Bacteriol. 189, 7932-7936. doi: 10.1128/JB.00693-07

Sawabe, T., Ogura, Y., Matsumura, Y., Feng, G., Amin, A. R., Mino, S., et al. (2013). Updating the Vibrio clades defined by multilocus sequence phylogeny: proposal of eight new clades, and the description of Vibrio tritonius sp. nov. Front. Microbiol. 4:414. doi: 10.3389/fmicb.2013.00414

Tatusova, T. A., and Madden, T. L. (1999). BLAST 2 SEQUENCES, a new tool for comparing protein and nucleotide sequences. FEMS Microbiol. Lett. 174, 247-250. doi: 10.1016/S0378-1097(99)00149-4

Thompson, C. C., Thompson, F. L., Vicente, A. C. P., and Swings, J. (2007). Phylogenetic analysis of vibrios and related species by means of atpA gene sequences. Int. J. Syst. Evol. Microbiol. 57, 2480-2484. doi: 10.1099/ijs.0.65223-0

Thompson, F., and Hoste, B. (2001). Genomic diversity amongst vibrio isolates from different sources determined by fluorescent amplified fragment length polymorphism. Syst. Appl. Microbiol. 538, 520-538. doi: 10.1078/0723-202000067
Thompson, F. L., Gevers, D., Thompson, C. C., Dawyndt, P., Hoste, B., Munn, C. B., et al. (2005). Phylogeny and molecular identification of vibrios on the basis of multilocus sequence analysis. Appl. Environ. Microbiol. 71, 5107-5115. doi: 10.1128/AEM.71.9.5107

Thompson, J. R., Randa, M. A., Marcelino, L. A., Tomita-Mitchell, A., Lim, E., and Polz, M. F. (2004). Diversity and dynamics of a north atlantic coastal Vibrio community. Appl. Environ. Microbiol. 70, 4103-4110. doi: 10.1128/AEM.70.7. 4103

Urakawa, H., Kita-Tsukamoto, K., and Ohwada, K. (1997). 16S rDNA genotyping using PCR/RFLP (restriction fragment length polymorphism) analysis among the family Vibrionaceae. FEMS Microbiol. Lett. 152, 125-132. doi: 10.1111/j. 1574-6968.1997.tb10418.x

Urakawa, H., Kita-Tsukamoto, K., and Ohwada, K. (1999). Restriction fragment length polymorphism analysis of psychrophilic and psychrotrophic Vibrio and Photobacterium from the north-western Pacific Ocean and Otsuchi Bay, Japan. Can. J. Microbiol. 45, 67-76. doi: 10.1139/cjm-45-1-67

Vezzulli, L., Brettar, I., Pezzati, E., Reid, P. C., Colwell, R. R., Höfle, M. G., et al. (2012). Long-term effects of ocean warming on the prokaryotic community: evidence from the vibrios. ISME J. 6, 21-30. doi: 10.1038/ismej. 2011.89

Xu, F., Ilyas, S., Hall, J. A., Jones, S. H., Cooper, V. S., and Whistler, C. A. (2015). Genetic characterization of clinical and environmental Vibrio parahaemolyticus from the Northeast USA reveals emerging resident and nonindigenous pathogen lineages. Front. Microbiol. 6:272. doi: 10.3389/fmicb.2015. 00272

Conflict of Interest Statement: The authors declare that the research was conducted in the absence of any commercial or financial relationships that could be construed as a potential conflict of interest.

Copyright (c) 2017 Machado, Cardoso, Giubergia, Rapacki and Gram. This is an open-access article distributed under the terms of the Creative Commons Attribution License (CC BY). The use, distribution or reproduction in other forums is permitted, provided the original author(s) or licensor are credited and that the original publication in this journal is cited, in accordance with accepted academic practice. No use, distribution or reproduction is permitted which does not comply with these terms. 